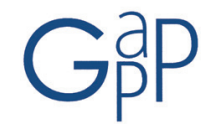

GESTIÓN Y ANÁLISIS DE POLÍTICAS PÚBLICAS, Nueva Época, n 10 julio-diciembre 2013 ISSN: 1989-8991

DOI: http://dx.doi.org/10.24965/gapp.voi10.10119

\title{
Las transformaciones de la política española de cooperación al desarrollo en gobernabilidad democrática: marco institucional, definición estratégica y acciones
}

\author{
Marisa Ramos Rollón \\ Conferencia de Ministros de los Países Iberoamericanos (COMJIB) \\ marisa.ramos@comjib.or
}

Recibido: 5 de noviembre de 2013

Aceptado: 13 de diciembre de 2013

Resumen

En este artículo se analiza la política española de cooperación al desarrollo en materia de gobernabilidad democrática, apuntando algunos rasgos de la trayectoria de la cooperación en este sector en la última década, y analizando los factores que explican las transformaciones de la misma, tanto los que se refieren a la evolución del marco institucional en el que se ha llevado a cabo, como al desarrollo estratégico y programático que la ha definido. Además, se analiza la evolución de los programas y proyectos en este ámbito. Este análisis tiene como objetivo contribuir a identificar cuáles han sido los cambios esenciales en la política de desarrollo en este sector, así como los factores que explican estos cambios y los límites de los mismos. Todo ello con el fin último de extraer aprendizajes del pasado más reciente que sirvan para mejorar las acciones a desarrollar en el futuro en este sector.

Palabras clave

Cooperación al desarrollo, gobernabilidad democrática, democracia, instituciones, desarrollo

\section{The Transformations of the Spanish Policy of Cooperation for the Development in Democratic Governance: Institutional Frame, Strategic Definition and Actions}

Abstract

This article analyzes the Spanish Policy of Cooperation for the Development in democratic governance, pointing some of the features of the cooperation track in this sector in the last decade, and also analyzing the factors that explain its own transformations, which concern both the evolution of the institutional frame where it has been executed, and to the strategic and programmatic development that sets it. Moreover, the evolution of the programs and projects in this area is analyzed. This analysis seeks to contribute to identify what were the essential changes in development policy in this sector, as well as the agents that explain these changes and the bounds of them. All of it with the purpose of drawing apprenticeship from the recent past which help to ameliorate the actions to develop hereafter in this sector.

Key words

Development cooperation, democratic governance, democracy, institutions, development 


\section{INTRODUCCIÓN}

El apoyo a la gobernabilidad democrática constituye uno de los sectores que caracteriza y da entidad a la política española en cooperación para el desarrollo desde sus inicios. Por diferentes factores, políticos, culturales, históricos o lingüísticos, España ha adquirido un rol de relevancia en cooperación al desarrollo precisamente por su contribución a este sector, especialmente orientada hacia la región latinoamericana. Diversos informes externos así lo acreditan, como el examen a la Cooperación Española de 2011 por parte del Comité de Ayuda al Desarrollo (CAD), que además insta a los actores centrales de esta cooperación a definir una mejor estrategia y desarrollar un mayor liderazgo en la ayuda internacional en el ámbito del desarrollo institucional, en áreas específicas como la reforma judicial, la descentralización, la administración fiscal o la reforma de los sistemas de seguridad y orientado hacia países de renta media'.

La trayectoria de la cooperación en este sector presenta, sin embargo, características particulares que afectan indudablemente al modelo de ayuda que se ha desarrollado y al impacto de la misma. España no solo ha alcanzado una posición reconocida en tanto país comprometido con el desarrollo a nivel internacional, sino que ha puesto a disposición de los países en desarrollo, especialmente hacia América Latina, su propia trayectoria de país que ha experimentado un profundo cambio político, económico y social, trayectoria que se suma al potencial que le aportan los lazos políticos, históricos y culturales que tiene con muchos de los países hacia los que se orienta esta ayuda. El apoyo de la cooperación española al sector de la gobernabilidad democrática se ha focalizado tradicionalmente en el fortalecimiento de ciertas instituciones públicas, fundamentalmente las relacionadas con el funcionamiento del estado de derecho, la administración pública y con los gobiernos locales. Las actuaciones que le han dado contenido han sido, además, básicamente técnicas y lideradas y gestionadas por entidades públicas españolas.

La cooperación en este sector ha estado liderada, fundamentalmente, por la Agencia Española de Cooperación Internacional para el Desarrollo (AECID)², en tanto que esta institución ha programado, diseñado, gestionado y/o coordinado la mayor parte de las actuaciones en materia de gobernabilidad democrática del conjunto de la Ayuda Oficial al Desarrollo (AOD) española.

La relevancia y prioridad que los distintos responsables políticos han dado a este sector, su consideración como ámbito que da entidad y define a la propia cooperación española y la complejidad de actores y subsectores que engloba, hacen del análisis de la ayuda en este sector un caso de interés para extraer conclusiones que permitan avanzar y mejorar en la forma de hacer cooperación al desarrollo. Y esto es especialmente necesario en un contexto de cambio y transformación de la política de ayuda al desarrollo, no solo en España, sino en el contexto internacional.

El objetivo de este artículo es apuntar algunos rasgos de la trayectoria de la ayuda en este sector en la última década, analizando tanto la evolución del marco institucional en el que se ha llevado a cabo, como el desarrollo estratégico y programático que la ha definido, y la evolución en el ámbito de la gestión de programas y proyectos en este ámbito. Este análisis tiene como objetivo contribuir a identificar cuáles han sido las transformaciones esenciales en la política de desarrollo en este sector, así como los factores que los explican y los límites de los mismos. Todo ello con el fin último de extraer aprendizajes del pasado más reciente que sirvan para mejorar las acciones a desarrollar en el futuro en este sector.

Para realizar este análisis, se cuenta con la información procedente de la experiencia de gestión de la autora en la AECID en el sector de la gobernabilidad democrática en América Latina entre los años 2005 y 2010 . Además, se ha contado con los datos procedentes de informes internos, como el Diagnóstico de la Ayuda de la AECID en el sector de gobierno y sociedad civil elaborado en noviembre de 2009 o el Informe sobre las intervenciones de cooperación en los sectores de gobernabilidad democrática y construcción de paz en América Latina (2008-2011). Esta información se complementa con la procedente de los Informes de Seguimiento del Plan Anual de la Cooperación Española ( $\mathrm{PACl})$, que ofrecen datos oficiales de gestión de la cooperación española en todos los sectores, incluidos los del sector de la gobernabilidad democrática.

En el ámbito teórico, la pregunta de investigación planteada, es decir, la descripción y explicación de los cambios y transformaciones de una política pública remite necesariamente a los análisis realizados sobre las ex-

\footnotetext{
1 Específicamente, el Informe recomienda: “Make capacity building a goal in its country partnership frameworks, and collect and share Spanish lessons and experience with capacity development, especially in middle-income countries” p. 20 OECD 2012.

2 La AECID se ha construido sobre la base de instituciones que la precedieron, en una suerte de sedimentación por etapas. Antes fue la Agencia Española de Cooperación Internacional (AECI), antes el Instituto de Cooperación Internacional (ICI) y antes incluso el Instituto de Cultura Hispánica (ICH).
} 
plicaciones del cambio de las políticas públicas, entre los que son referencia ineludible los trabajos Hogwood and Peters (1983), Sabatier y Jenkins (1993) o Capano and Howlett (2009).

En el análisis que se plantea en este trabajo se hace especial énfasis en dos aspectos nucleares para explicar las transformaciones en las políticas públicas: la relación entre estrategia y acción o los denominados problemas de implementación y los análisis de actores, especialmente en lo que se refiere a coaliciones promotoras. Ambos planteamientos teórico-metodológicos resultan de especial utilidad para analizar la política objeto de análisis en este artículo. En el primer caso, encaja especialmente bien la complejidad de la relación entre estrategias y acción con la política, planteada por algunos autores como Mintzberg y Jorgensen (1995), que establecen la pertinencia de analizar el proceso de formulación e implementación superando el modelo racional o del actor inteligente y las críticas y cuestionamientos realizados a esa modelo que representa el incrementalismo de Lindblom (1992). Mintzberg y Jorgensen (1995) sitúan en lugar central de su análisis la idea de la estrategia, en tanto norma de acción congruente en el comportamiento a través del tiempo, diferenciando además entre estrategia deliberada, equivalente al concepto de planificación y formulación del modelo racional y la estrategia emergente, que incorpora la idea de aprendizaje, en tanto que las organizaciones pueden aprender mientras formulan la estrategia.

Desde los años 80 , la cuestión teórica relativa al cambio en las políticas públicas tuvo un impulso importante con los trabajos críticos al modelo tradicional del ciclo de políticas y la propuesta alternativa de las coaliciones promotoras desarrollados por Sabatier y Jenkins-Smith (1993). Estos autores consideran necesario desechar el modelo del ciclo por considerarlo poco útil para tomar en cuenta la realidad de los procesos políticos y permitir explicar los cambios de las políticas a largo plazo (Sabatier, 1999). El origen del enfoque de las coaliciones promotoras se plantea, según su propio proponente (Sabatier, 1999: 117-120) con la intención de desarrollar una alternativa al enfoque del ciclo de las políticas entonces dominante, con el fin de realizar una síntesis de los enfoques top down y bottom up en el estudio de la implementación e integrar de manera más satisfactoria el papel de los técnicos y otros actores en los procesos de política.

Estos enfoques resultan especialmente útil para abordar la política española de cooperación al desarrollo en general, ya que ésta ha sido una política especialmente prolija en fundamentaciones teóricas y planteamientos estratégicos cuya implementación y ejecución ha sido especialmente compleja y en la que se ha puesto de manifiesto la dificultad para integrar estrategia y acción, para integrar en la estrategia el aporte técnico y en definitiva para facilitar el aprendizaje en la formulación e implementación de esta política.

\section{GOBERNABILIDAD DEMOCRÁTICA Y DESARROLLO}

Las instituciones no fueron centrales en el debate sobre el desarrollo hasta que el enfoque neoinstitucionalista en la década de 1970 rescató la necesidad de vincular el desarrollo con las instituciones. A partir de ese momento, se generó un cambio importante en la evolución de la economía, la ciencia política y la sociología promoviéndose una convergencia de sus enfoques en temas como las instituciones y el desarrollo económico. Esta convergencia giró alrededor del concepto de "instituciones", un término familiar en sociología y antropología social pero que constituyó un cambio importante en las teorías del desarrollo, dominadas hasta ese momento por el paradigma neoclásico y especialmente por las teorías de la modernización. Sin embargo, esta convergencia ha ido acompañada de bastante confusión conceptual y teórica, que requiere un análisis del concepto de instituciones y su vinculación con las formas políticas vinculadas a la democracia.

El concepto de institución se desarrolla en el ámbito de las teorías del desarrollo. Según el neoinstitucionalismo, para entender el funcionamiento social y económico es necesario entender las reglas del juego, las normas que rigen las interacciones de los actores, normas que pueden ser formales pero también informales. Este concepto de institución incide directamente, por tanto, en el crecimiento económico y por ende en el desarrollo, ya que las reglas del juego marcan los límites de actuación y el comportamiento de los actores, y por tanto, de las fuerzas sociales y económicas. Más aún, no solo limitan ese comportamiento sino que moldean la propia configuración de actores. A partir del trabajo de North (1990), son numerosos los trabajos que constituyen ya una referencia en el establecimiento de los vínculos entre desarrollo e instituciones políticas (Kaufmann y otros, 1999, Rodrik y otros, 2004 o Grindle, 2007, entre otros) y entre desarrollo y democracia (Diamond, 1993 y Przeworski, 2004).

Las instituciones políticas, por tanto, explican y condicionan el desarrollo, y su diseño y eficacia tienen una implicación directa en el mismo. Su adecuado funcionamiento y su mejora es, consecuentemente, un requisito, una condición para el desarrollo. La idea de promover eficacia en las instituciones como instrumento al mejor funcionamiento del mercado se traduce en la necesidad de promover la gobernabilidad como instrumento para promover desarrollo. 
El concepto de desarrollo humano (Sen, 1989) aporta una visión mucho más amplia a la idea de desarrollo, superando las visiones más constreñidas del desarrollo que lo equiparaban al crecimiento económico. Incorpora en la propia idea de desarrollo la garantía y protección de derechos, no solo los derechos humanos, sino también los políticos y económicos. Desde esta óptica, la protección y garantía de los derechos por parte de las instituciones forma parte intrínseca del propio concepto de desarrollo. La idea de democracia por tanto se integra como una dimensión más del desarrollo humano. El funcionamiento adecuado de las instituciones para protegerlos y garantizarlos ya no es una vía al desarrollo, sino que es una vertiente misma del desarrollo.

Estas revisiones conceptuales y teóricas se han plasmado en las políticas de cooperación al desarrollo desde hace unas décadas, de forma que ya todas las agencias y actores que promueven desarrollo incorporan entre sus prioridades el apoyo a la gobernabilidad, ahora ya con el adjetivo de democrática. El vínculo entre democracia, tanto en lo que se refiere a derechos como a inclusión y participación, y desarrollo se plasma en programas y proyectos concretos que dan contenido a las políticas de desarrollo en las últimas décadas. En este proceso no fue ajena la Cooperación Española.

\section{TRAYECTORIA DE LA COOPERACIÓN ESPAÑOLA EN EL SECTOR DE LA GOBERNABILIDAD DEMOCRÁTICA EN LA ÚLTIMA DÉCADA}

\section{La evolución del marco institucional de la cooperación en este sector}

España comenzó a desarrollar un papel como país donante a finales de la década de 1980 cuando asumió un compromiso político de primer orden con los procesos de paz en Centroamérica y con la resolución del conflicto armado en esa región (Díaz Rato, 2007: 349 y ss.). Sin duda, ese fue el inicio de lo que podría denominarse política de cooperación al desarrollo, aunque en estos años la ayuda se llevó a cabo de acuerdo a unos patrones sui generis, de difícil encaje, en la mayor parte de los casos, en los parámetros esenciales de la ayuda al desarrollo a nivel internacional, definida fundamentalmente por el Comité de Ayuda al Desarrollo (CAD).

La conformación del Instituto de Cooperación Iberoamericana (ICI) en torno a la Conmemoración del V Centenario otorgó a esta política la estructura institucional necesaria para encajar las acciones y para desarrollar esta incipiente política pública de compromiso con el desarrollo. La creación de las primeras oficinas en el exterior se orientaron en buena medida hacia el apoyo a diversos procesos políticos, acompañados de otros programas sociales más o menos insertables en lo que ahora se denomina ayuda al desarrollo.

En cualquier caso, el apoyo al sector de gobernabilidad en la región centroamericana da, en buena medida, origen y entidad a la política de ayuda al desarrollo en su conjunto, de forma que a partir de este germen se empiezan a llevar a cabo acciones en gran parte de los países de la región latinoamericana así como en otras áreas geográficas, principalmente el Magreb. Y todo ello en un momento en el que ya se amplían los sectores de intervención hacia ámbitos como el patrimonio, la educación o la salud.

A finales de la década de 1980 se produce un crecimiento importante de la política de cooperación al desarrollo en su conjunto, sobre todo la que lleva a cabo el ICI luego denominado AECI, constituido como tal en 1988. Se inicia un despliegue de estructura en la mayor parte de los países de la región y se inician programas de cooperación amplios y diversos, siendo la gobernabilidad democrática un sector constante y permanente en todos ellos. La década de 1990 supone la puesta de largo de la política española de cooperación al desarrollo y el tránsito decisivo de España como país receptor de fondos de desarrollo a país donante de ayuda al desarrollo. No hay que olvidar que tal como señala Robles (2007: 73) España solicitó dejar de ser parte de la lista del Comité de Ayuda al Desarrollo (CAD) de países en desarrollo en 1983 y no fue miembro de dicho Comité hasta 1991, es decir, hace poco más de 20 años. En 1998 se aprueba, con acuerdo de todos los grupos, la Ley de Cooperación Internacional, que trató de dotar de principios, objetivos, prioridades y modalidades a esta política. Se le otorgaba a la AECI la gestión fundamental de la política. Así mismo, se crearon otros órganos, como la Comisión Interministerial (2000) o el Consejo de Cooperación (2001).

En la década de 2000 se inicia un proceso de crecimiento importante de la propia $\mathrm{AECl}$, de forma que se consolida el despliegue de su estructura en América Latina y el norte de África y adquieren relevancia proyectos en este sector en la mayor parte de los países. Es también el momento en el que se inician programas de ámbito regional, como el Programa de Cooperación Regional con Centroamérica (PCRC), que aunque incluye algunas acciones en gobernabilidad democrática, se orienta fundamentalmente a otros ámbitos relacionados con sectores sociales básicos y productivos (educación, empleo o turismo). La cooperación en este sector se llevó a cabo, además, de la mano e, incluso, orientada por ciertas instituciones españolas y por tanto, induciendo ciertos temas y modos de 
gestión. Estos rasgos son especialmente pertinentes para los subsectores de justicia y seguridad, de administración pública y de administración local³.

Sin embargo, a lo largo de la década de 2000, la cooperación en este sector aportó una experiencia para el conjunto de las instituciones españolas competentes en la política de ayuda al desarrollo que resultó decisiva sobre todo para potenciar y/o profundizar lazos entre instituciones y para facilitar el diálogo y la interlocución política, que capitalizaron fundamentalmente las mismas instituciones públicas españolas que estaban tras el diseño y la gestión de los proyectos y programas, como el Consejo General del Poder Judicial (CGPJ), el Ministerio del Interior o la Agencia Tributaria. Esta capacidad de interlocución no repercutió en la misma medida en la propia $\mathrm{AECl}$, que la mayoría de las veces quedaba reducida a la función de caja pagadora de las acciones. Tampoco fue orientada y/o rentabilizada como habría sido deseable por parte de la política exterior española, más allá de la utilidad práctica, a veces no menor, que le otorgaba a las embajadas la posición de donante flexible y confiable. Por tanto, esta cooperación acabó quedando reducida a actuaciones y apoyos de carácter puntual, basados en una relación oferta-demanda poco teorizada, poco ordenada y, por tanto, con impactos relativamente bajos. A su vez, se llevó a cabo de forma muy fragmentada, poco orientada y muy dependiente de elementos coyunturales y/o de intereses de las entidades públicas españolas que las gestionaban, en colaboración con las contrapartes locales, así como, a veces también del interés de la política exterior del momento.

A partir de 2004-2005, se producen una serie de cambios que se reflejan en un importante crecimiento presupuestario, en la ampliación de nuevas áreas geográficas a las que orientar la ayuda y en la incorporación de los principios emanados de los debates desarrollados en el seno de la comunidad donante del ámbito OCDE, sobre todo en lo que se refiere a la adopción de los principios relacionados con la calidad de la ayuda. La Declaración de París de 2005 se convierte en el planteamiento orientador de las acciones de la cooperación española a partir de ese momento (Iglesias Caruncho y otros, 2007: 13-14). Con estos cambios, la política de cooperación española asumiría la ortodoxia avanzada en el ámbito de la comunidad internacional y España pasaría a realizar un esfuerzo de gigante por dejar de ser un país donante sui generis y particular en sus orientaciones, zonas de interés y principios, para convertirse en un país "normal” (Ayuso, 2007: 565-568) en lo que se refiere a los principios estandarizados por la comunidad donante. De hecho, los principales cambios de esta etapa, crecimiento presupuestario, cambio institucional, incorporación de nuevas áreas y nuevos instrumentos y fortalecimiento de la planificación y la teorización pueden ser interpretados en esta clave, en la necesidad de que España asumiera la ortodoxia en esta política y con ello superar su particularidad como país donante.

Además, estos cambios se plasmaron en las propuestas de cambio de la $\mathrm{AECl}$, como principal actor público de esta política. Este cambio se reflejó en el crecimiento de la organización, que en ningún caso fue parejo al incremento del presupuesto a gestionar. Este cambio se plasmó en el Real Decreto 1403/2007, de 26 de octubre, según el cual se reguló la nueva Agencia Española de Cooperación para el Desarrollo (AECID), añadiéndole al nombre de la institución el término Desarrollo. Igualmente, se materializó en la apertura de nuevas oficinas, en la creación de nuevas unidades en la sede en Madrid y en la adopción de procedimientos supuestamente más ágiles en el marco de la ley de agencias de 2001 que sirvió de cobertura. De igual manera, se incorporó en la estructura de la nueva Agencia la transversalización del enfoque sectorial, de forma que se creó, con rango de Dirección General, una unidad administrativa para trabajar el ámbito sectorial, incluyéndose en ella, como un sector más, la gobernabilidad democrática4.

\section{El énfasis en la definición del marco estratégico y programático}

Indudablemente, uno de los principales cambios que caracterizaron esta última etapa, desde 2004, fue el peso otorgado a la definición de un marco teórico y de una estrategia de la política de cooperación al desarrollo, que se reflejó en la transformación de la anterior Oficina de Planificación y Estrategia (OPE) en la Dirección General de Políticas de Desarrollo (DGPOLDE), multiplicando sus medios, presupuestarios y humanos. En ese escenario se comenzó a elaborar un entramado teórico y programático, en el que se elaboraron algunos documentos clave para el sector de la gobernabilidad democrática5. Hay que resaltar que el esfuerzo de definición estratégica se ha-

3 Por supuesto, que en esta época se iniciara el control del sector por parte de ciertas instituciones públicas españolas, como el Consejo General del Poder Judicial o por parte de ministerios o entes locales dependió de factores diversos, pero en ningún caso respondió a un análisis riguroso de cuáles eran los ámbitos en los que España tendría un valor añadido. Específicamente en justicia, se da la paradoja de que se ofertó apoyo técnico en sectores en los que España no tiene mucho que aportar, más bien todo lo contrario, como por ejemplo en gestión o gobierno de la justicia.

4 Hay que destacar que el enfoque sectorial no resultó tan novedoso en este sector pues ya había una experiencia previa de incorporación del enfoque sectorial en gobernabilidad en el ámbito geográfico de América Latina. Paradójicamente, el rango otorgado a este sector en la nueva unidad de cooperación sectorial fue menor que el otorgado a otros sectores.

5 Estos documentos fueron fundamentalmente los Planes Directores de la Cooperación Española de 2005 -2008 y $2009-2012$ así como varias Estrategias sectoriales y geográficas.

GESTIÓN Y ANÁLISIS DE POLÍTICAS PÚBLICAS, Nueva Época, n 10 julio-diciembre 2013 ISSN: 1989-8991 - DOI: 10.24965/gapp.voi10.10119 
bía iniciado a finales de los años noventa y que en los primeros años de la década de 2000 ya se habían elaborado documentos clave para el sector de la gobernabilidad democrática.

En primer lugar, la ley 23/1998 de 7 de julio de Cooperación Internacional para el Desarrollo, en su artículo 3, ya reconocía el apoyo a la instauración y consolidación de regímenes democráticos y el respeto a los derechos y libertades fundamentales, como elementos centrales de la política de cooperación al desarrollo. En su artículo 7 incluía, asimismo, como uno de los objetivos de la Cooperación Española "el fortalecimiento de las estructuras democráticas y de la sociedad civil y el apoyo a las instituciones, especialmente las más próximas al ciudadano". Posteriormente, el I Plan Director establecía como objetivos sectoriales la promoción de la participación social, el desarrollo institucional y el buen gobierno, concretándose en la defensa de los derechos humanos y el apoyo al estado de derecho, en la promoción de la participación social, en el desarrollo institucional y en las prácticas de buen gobierno, incluyendo el establecimiento del marco regulador económico y financiero adecuado y la solvencia de las políticas públicas. Como desarrollo de estos enunciados, en 2001 se elaboró una Estrategia para la Promoción de la Democracia y el Estado de Derecho, que se proponía ofrecer un marco de coordinación entre actores españoles públicos y privados para aumentar la coherencia y la eficacia de sus acciones en materia del apoyo a la gobernabilidad democrática. Con el fin de facilitar el aterrizaje de esta Estrategia en la región iberoamericana, se elaboró un segundo documento programático, el Plan de Fortalecimiento de las Instituciones Democráticas en Iberoamérica que adoptó un enfoque sectorial en materia de gobernabilidad democrática, al tiempo que proponía un diagnóstico y un marco de intervención en este ámbito para la región.

El II Plan Director de la Cooperación Española de 2005 marcó un punto de inflexión en la política de cooperación al desarrollo en general. Este II Plan Director se constituyó en la declaración de intenciones de los ambiciosos cambios que se estaban llevando a cabo en la política de cooperación al desarrollo. Sin embargo, este II Plan Director fue parco en la parte relativa a la ayuda en el sector de la gobernabilidad democrática, denominada en ese momento gobernanza democrática. La prioridad sectorial de apoyo a la gobernanza democrática, la participación ciudadana y el desarrollo institucional que estableció el II Plan Director se apoyaba en una serie de premisas orientadas, teóricamente, por el concepto de gobernanza, de forma que no sólo se limitaba a la promoción de la incidencia en el ámbito político-institucional para conseguir instituciones sólidas que se convirtieran en el pilar de un crecimiento económico con justicia y equidad, sino también en el fortalecimiento de los espacios de consenso entre diversos actores privados y públicos, nacionales e internacionales, para la toma de decisiones públicas orientadas al desarrollo y al empoderamiento de las personas. Específicamente, se marcaron una serie de líneas estratégicas, tales como la promoción de la democracia representativa y participativa, y del pluralismo político, el apoyo y el fortalecimiento de los procesos y de los mecanismos institucionales del diálogo social, el fortalecimiento del Estado de Derecho, el desarrollo de la Administración al servicio del ciudadano y la buena gestión de los asuntos públicos y la descentralización y fortalecimiento de las administraciones locales. En términos generales, la prioridad sectorial otorgada a la gobernabilidad democrática, no se elaboró de forma precisa y concreta, sino que se expresó a través de planteamientos muy generales y por tanto de difícil aplicación en forma de acciones concretas.

Tratando de superar este límite, se elaboró posteriormente una Estrategia de Gobernabilidad Democrática, Participación Ciudadana y Desarrollo Institucional que buscaba orientar de forma más concreta y precisa las acciones que deberían llevarse a cabo en este sector. Se planteó un doble enfoque: fortalecimiento institucional y apoyo a las reformas, y fortalecimiento de la ciudadanía desde el reconocimiento de los derechos sociales, civiles y políticos. La estrategia marcaba como objetivo general la promoción de la calidad de la democracia y el respeto de los derechos fundamentales desde una participación real y efectiva de la ciudadanía y como objetivos concretos el fortalecimiento de las administraciones públicas que mejoraran la cohesión social, el fortalecimiento de la seguridad pública, el acceso a la justicia y la promoción de los derechos humanos y la promoción de la descentralización y la participación ciudadana. Sin embargo, esta Estrategia mantuvo un nivel de generalidad e imprecisión similar al del Plan Director.

La Estrategia optó por el enfoque de derechos como eje de la cooperación española en el sector, enfoque que resultaba absolutamente coherente con la idea de desarrollo humano que ya para entonces guiaba la cooperación en general y con la centralidad otorgada al individuo como portador de derechos. Sin embargo, el enfoque no se precisaba en términos operativos, de forma que se mezclaron conceptos y argumentos, sin definir siquiera claramente lo que se entendía por gobernanza democrática, por participación ciudadana, por desarrollo institucional o por cohesión social, entre otros. La Estrategia situaba la "redefinición de las relaciones de poder" (p. 6) como el punto central del enfoque de derechos, premisa que presenta varios problemas. En el plano operativo, resulta complejo determinar cuáles son las relaciones de poder que son "idóneas" para el desarrollo; en el ámbito sustantivo, la idea de "redefinir la relaciones de poder" sitúa el debate en la dicotomía entre el apoyo a los gobiernos con el fin de promover la alineación de políticas y el apoyo a la sociedad civil como estrategia de garantía y protección 
de derechos, debate que va más allá de la diferencia clásica entre Estado y sociedad a las que continuamente hacía referencia la Estrategia. En este mismo sentido, la Estrategia pretendía superar el enfoque institucionalista, cuestionando incluso los efectos que tiene en el desarrollo el fortalecimiento institucional en contextos de baja ciudadanía (p. 9). Todo ello sin definir de forma precisa el significado del término institución, equivalente en el documento unas veces a norma o regla formal, otras a gobierno $0^{6}$, y otras a la idea de Estado en contraposición a la de sociedad. Tampoco resultó muy operativo diferenciar entre instituciones formales e informales, sobre todo cuando en estas últimas se incluyen conceptos tan amplios como "la seguridad privada, la migración o la costumbre para la administración de justicia".

En 2009, el III Plan Director de la Cooperación Española 2009-2013, de nuevo marcó este sector como uno de los prioritarios, identificando como objetivo esencial del mismo la promoción de la calidad de la democracia y respeto de los derechos fundamentales desde una participación real y efectiva de la ciudadanía, el ejercicio de los derechos humanos y las capacidades para promover el desarrollo.

El III Plan Director también apostó por potenciar ciertos cambios que podrían afectar específicamente al sector de la gobernabilidad democrática. Por una parte, se consideró necesario concentrar más el apoyo hacia ciertos subsectores clave, desde la convicción de que hay ámbitos de la institucionalidad democrática que tienen un impacto más directo en la extensión de la ciudadanía, en el reconocimiento de derechos básicos y en la capacidad para hacerlos efectivos, como es el apoyo al sistema democrático y al fortalecimiento de derechos y a la promoción de capacidades para desarrollar políticas públicas de desarrollo a través de administraciones públicas inclusivas y eficientes.

El III Plan Director identificó la gobernabilidad democrática y los derechos humanos como prioridades horizontales, "al ser elementos cruciales a tener en cuenta en la planificación, ejecución, seguimiento y evaluación de la Cooperación Española". Asimismo, promueve el sector de la gobernabilidad democrática en tanto que ámbito de reconocimiento del derecho de las personas a tener un desarrollo sostenible. Esta apuesta por la gobernabilidad no precisa de forma suficiente, sin embargo, ámbitos específicos de intervención, ya que la concibe como un apoyo a "las instituciones para garantizar el acceso a determinados servicios básicos, los derechos políticos y la igualdad de género". En este sentido, se apunta que la gobernabilidad tiene diversas dimensiones, tales como: desarrollo institucional, participación ciudadana, promoción de la igualdad, promoción de la cohesión social, rendición de cuentas, etc. Estos aspectos se engloban en una doble dirección: a) mejora y reforma de la institucionalidad democrática, y b) apoyo a la organización de la sociedad civil.

Intentando precisar, el mismo III Plan Director apunta algunos elementos clave de la gobernabilidad democrática. En el ámbito de la calidad democrática, la construcción de un Estado basado en el Derecho, la división de poderes conforme a derecho y la vinculación de demandas ciudadanas e instituciones. $Y$ en lo que se refiere al crecimiento económico distributivo, redistribución del ingreso. No se llegó a desarrollar una Estrategia en este sector que especificara lo planteado de forma más genérica y global en el Plan Director, así como tampoco se elaboró un Plan de Actuación Sectorial, mientras que sí se hizo en otros sectores como Medio Ambiente, Agua, Género o Salud.

Sin embargo, todo este entramado programático, el II Plan Director, la Estrategia y el III Plan Director, resultó muy genérico y vago en este sector, de forma que no se aportaron herramientas adecuadas que dieran las suficientes pistas acerca de cómo concretar y precisar en programas y acciones concretas, de identificación y selección de socios y actores o de ubicación de niveles preferentes de intervención. Aunque las Estrategias Sectoriales pretendieron orientar y definir estrategias de acción, la generalidad y la exhaustividad en actores y subsectores volvieron a dejar sin definición y sin priorización un sector que ya es en sí mismo amplio, ambiguo y vago. Consecuentemente, se dejó al voluntarismo político, a la toma de decisiones fragmentada, a la casuística de cada contexto y a la inercia, la adopción y gestión de programas y proyectos en este ámbito, como claro ejemplo de las dificultades de combinar la estrategia y la acción que plantean MIntzburg y Jorgensen (1995).

Este hecho podría ser explicado por la débil incorporación de la experiencia previa acumulada en este sector al proceso de reflexión y elaboración programática y estratégica. Se podría señalar que esta reflexión estratégica no utilizó como fuente de inspiración la experiencia acumulada en la gestión de programas específicos, sino que se elaboró a partir de la doctrina imperante, poniendo de manifiesto las dificultades para incorporar las facetas técnicas en la formulación de políticas, en la estrategia. Tal como ha señalado Alonso "este deficiente resultado

6 No creo que sea necesario hacer referencia a la amplísima corriente del neoinstitucionalismo y a la concepción amplia de institución que desde el trabajo seminal de North (1991) considera a las instituciones como reglas del juego entre actores. 
apunta a un problema más general de la cooperación española: su limitada capacidad para integrar la reflexión doctrinal y estratégica en la práctica efectiva y en la gestión diaria de su sistema de cooperación y viceversa. Como una especie de sistema esquizoide, unos son los que generan pensamiento estratégico y otros son los que ejecutan y gestionan" (Alonso, 2007: 168).

\section{La evolución en la gestión de programas y proyectos en el sector de la gobernabilidad democrática}

La ayuda de la $\mathrm{AECl}$ en materia de gobernabilidad creció en esos años, tal como crecieron el resto de sectores. Aunque los únicos datos disponibles son los del PACl, sobre esa base se pueden destacar algunas tendencias. En primer lugar, el porcentaje del presupuesto que se destina al sector de gobernabilidad gira en torno a un $18 \%$ del total del presupuesto de la AOD, siendo ligeramente creciente de 2005 a 2009 y bajando de forma significativa a partir de ese año (17,8 en 2006, 18,10 en 2007, 20,0 en 2008, 23,8 en 2009 y 19,04 en 2010) (Figura 1 ).

\section{FIGURA 1}

\% DE AOD DESTINADO AL SECTOR DE GOBERNABILIDAD DEMOCRÁTICA

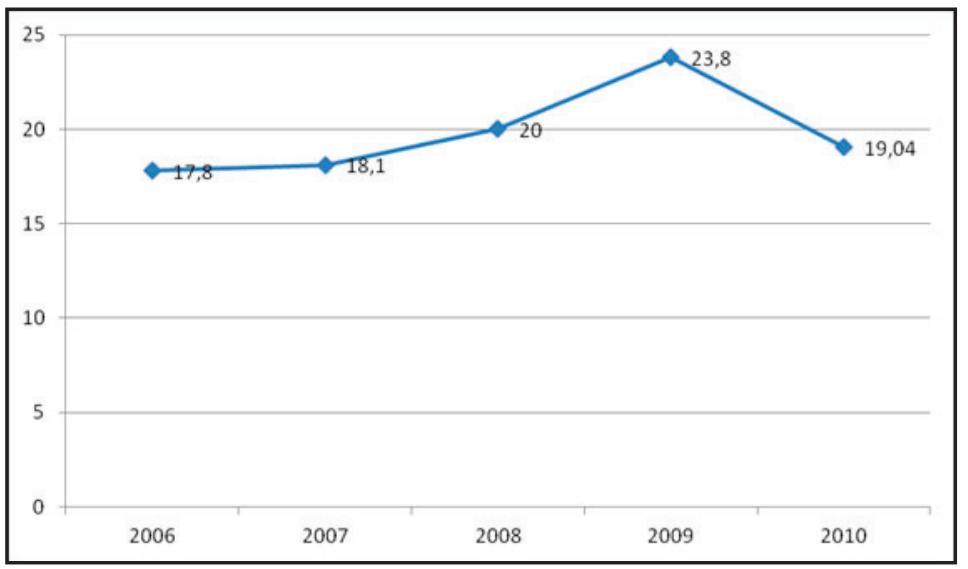

FUENTE: Seguimiento PACI

De acuerdo con los datos procedentes del Diagnóstico elaborado en la AECID en 2009 sobre este sector, la ayuda de la AECID en el ámbito de la gobernabilidad en el periodo 2005-2008 ascendió a un total de 366,48 millones de euros ${ }^{7}$, lo que representaba alrededor de un $14,7 \%$ de la AOD total proporcionada desde la AECID en el mismo periodo. El sector de gobernabilidad experimentó un crecimiento muy significativo en el periodo, pasando de 25,7 M€ en 2005 a 162 M€ en 2008. La cooperación creció en todas las modalidades, aunque la realizada por vía bilateral fue perdiendo importancia relativa en relación con las actuaciones ejecutadas por organismos internacionales.

De acuerdo con los datos que arroja el Diagnóstico, puede plantearse que casi dos tercios del total de la ayuda en gobernabilidad de estos años se dirigieron al fortalecimiento de capacidades de las administraciones públicas e instancias del Estado ${ }^{8}$, mientras que alrededor de un $30 \%$ se dirigió al fortalecimiento de entidades de la sociedad civil9. Esto significa que se siguió manteniendo el patrón fundamental de cooperación de la AECID en materia de gobernabilidad, orientándose la misma al fortalecimiento de las instituciones públicas de los países destinatarios.

También resulta de interés conocer la vía o modalidad a través de la cual se lleva a cabo la ayuda en el sector de la gobernabilidad. Más de la mitad del volumen total en el periodo 2005-2008 se distribuyó por vía bilateral, mientras que un $44 \%$ se canalizó a través de organismos multilaterales de desarrollo. Una parte importante del crecimiento presupuestario del sector en el periodo 2005-2008 descansó en la cooperación multilateral y multibilateral. En efecto, mientras el volumen total de cooperación bilateral en gobernabilidad se cuadriplicó prácticamente entre 2005 y 2008, la cooperación multibilateral se multiplicó por 11 y la multilateral por 258. Si la cooperación bilateral representaba en 2005 el 80\%, en 2008 esa proporción descendió al 47\%.

7 Esta cifra no incluye las intervenciones en el ámbito de la descentralización y fortalecimiento municipal, ni las actuaciones en el ámbito de construcción de paz y prevención de conflictos.

8 (subsectores 15110, 15120, 15130, 15140, 15161, 15210).

$9(15150,15162$ y 15163$)$ 
Esta tendencia puede explicarse por la limitada capacidad de gestión de la AECID para asumir por vía bilateral un crecimiento tan significativo de recursos en tan poco tiempo, delegándose esa gestión a terceros organismos teóricamente especializados. Pero además resultó consistente con la apuesta del Plan Director 2005-2008 por aumentar los recursos destinados a la cooperación multilateral, en especial a favor del apoyo a los esfuerzos para el cumplimiento de los Objetivos del Milenio (ODM).

Por regiones, América Latina continuó siendo la principal destinataria de los recursos de la AECID en gobernabilidad. Centroamérica y Caribe (24\% del total), América del Sur (15\%) y América no especificados (15\%) aglutinan alrededor del 54\% de la AOD en el sector. Le siguen por este orden África del Norte y Oriente Medio (13\%), África Subsahariana (10\%), Asia Pacífico (7\%) y Europa Central y Oriental (3\%), (Figura 2).

FIGURA 2

\section{DESTINO DE LOS RECURSOS AECID EN SECTOR DE GOBERNABILIDAD DEMOCRÁTICA}

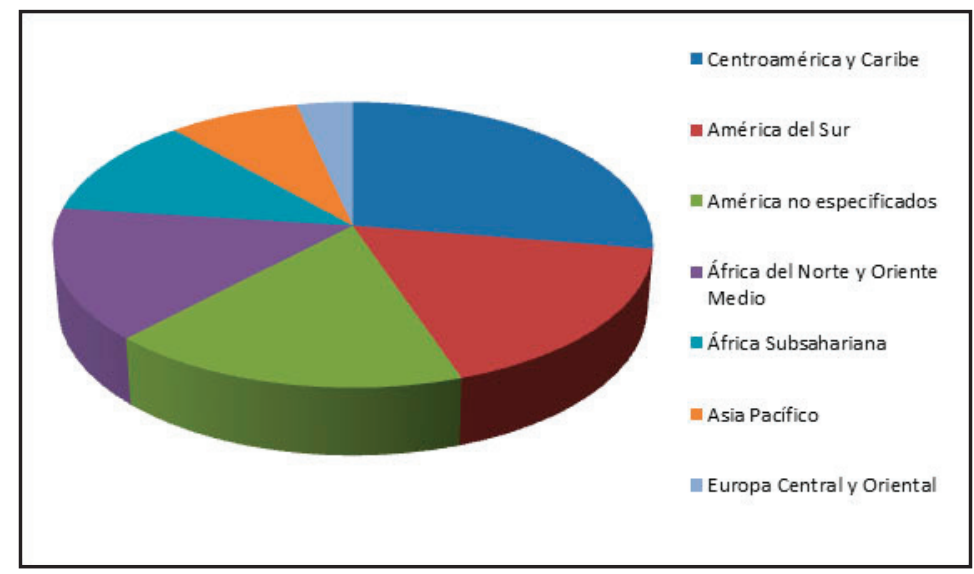

FUENTE: Diagnóstico Gobernabilidad Democrática

En lo que se refiere a los instrumentos, prácticamente la mitad (48\%) de los recursos de cooperación de la AECID en el ámbito de la gobernabilidad se ejecuta a través del instrumento “programa/proyecto". Le siguen en importancia el fondo común/cesta de donantes/contribución a OOII (30\%), la ayuda programática - apoyo presupuestario o enfoque sectorial (6\%), los cursos y seminarios (6\%) y las asistencias técnicas puntuales (5\% del total). En relación con los instrumentos de financiación en el sector, el más utilizado fue la transferencia a OOII, con aproximadamente el 29\% del total. A la par se encuentran las Subvenciones de Estado bilaterales (29\% de los fondos), seguida por las subvenciones a ONGD (9\%), Subvención de Estado Multilateral (8\%), la Convocatoria Abierta y Permanente (CAP, 4\%) y la Subvención nominativa (3\%). Los contratos de servicios apenas aparecen como mecanismo de financiación. Cabe señalar que no se pudo determinar el instrumento de financiación para el 18\% del presupuesto.

Se constata que hay un elevado número de intervenciones de gobernabilidad en el periodo 2005-2008 por país. En algunos países (Colombia, Guatemala, Perú), se realizaron entre 40 y 60 intervenciones en gobernabilidad, lo que revela una muy escasa concentración de esfuerzos. La información disponible es bastante completa en relación con las entidades ejecutoras de las intervenciones en el sector, pero no se dispone de información suficiente en relación con las entidades contraparte (socios locales).

En cuanto a la participación de entidades españolas como ejecutoras de las intervenciones de gobernabilidad (al margen de la ejecución directa por AECID en los Centros de Formación), por volumen de fondos destaca la FIIAPP, el Instituto Nacional de Administración Pública (INAP), el Instituto de Estudios Fiscales y el Consejo General del Poder Judicial entre las instituciones de carácter público, y ACSUR, Humanismo y Democracia y Solidaridad Internacional entre las de carácter privado. Globalmente en un 33\% de las intervenciones bilaterales de cooperación en gobernabilidad han estado presentes entidades españolas como ejecutoras. Desde el punto de vista presupuestario han ejecutado el 38\% del presupuesto total bilateral. Si tenemos en cuenta la totalidad de las intervenciones en el período (no sólo las bilaterales), en un $26 \%$ de ellas han estado presentes entidades españolas, que a nivel presupuestario representan un $20 \%$ del total. Por último, si, debido a su singularidad, excluimos de los cálculos las intervenciones gestionadas por la FIAPP, los porcentajes bajan al 19\% del total en número de intervenciones y al $17 \%$ en presupuesto, gestionado por entidades españolas. Por otra parte, se constata además que dentro de las intervenciones ejecutadas por entidades españolas, aproximadamente el $37 \%$ lo fueron por entidades públicas. 
De acuerdo con todo ello, se puede considerar que el crecimiento presupuestario en el sector de gobernabilidad democrática en este periodo fue importante pero menor que el crecimiento global en la AOD y menor que en otros sectores. El principal cambio desde la perspectiva cuantitativa lo constituye la canalización de parte de los recursos hacia fondos multilaterales, manteniéndose un patrón de gestión basada en la cooperación bilateral, especialmente hacia los países de América Latina y con importante participación de entidades públicas españolas en la gestión directa.

Sin embargo, sí se pueden identificar cambios más sustanciales desde una mirada más cualitativa, indagando en los modelos de gestión de los programas y proyectos llevados a cabo durante este periodo. Estos cambios se podrían resumir en los tres siguientes:

- Promoción de la AECID como actor central en la toma de decisiones y en la definición de la política de cooperación al desarrollo del gobierno central, potenciando su capacidad de coordinación con otros actores públicos españoles (CCAA, ONGD y EELL).

- Diversificación de los socios españoles para la ejecución de las acciones, rompiendo con la exclusiva que habían tenido ciertos actores e instituciones hasta entonces (fundamentalmente el CGPJ en el sector justicia o el Ministerio del Interior en el sector seguridad).

- Ampliación de los temas y de los ámbitos geográficos de intervención de acuerdo a una lógica más amplia de oferta y demanda.

Estos tres cambios se vieron facilitados por el crecimiento presupuestario antes mencionado, que permitió ampliar el número de socios y de sectores de intervención sin sacrificar de forma dramática el trabajo con los ya existentes. Además, estas transformaciones no se corresponden, de forma nítida, con un cambio de orientación ideológica, sino más bien con la decisión de hacer de España un donante relevante en el panorama internacional. No se hizo una adecuada labor de aprendizaje basada en la experiencia acumulada (se han realizado muy pocas evaluaciones en este sector) y no se identificaron de forma rigurosa las lecciones aprendidas; más bien al contrario, se promovió un discurso negativo y descalificador de gran parte de la cooperación realizada en este sector.

\section{LOS FACTORES EXPLICATIVOS DE LAS TRANSFORMACIONES}

Las transformaciones mencionadas se explican, en parte, por una serie de factores derivados, fundamentalmente, de la coyuntura política caracterizada por la situación de crecimiento económico, sobre todo en la etapa 2004 a 2008 y por la orientación ideológica del gobierno, que situó la política cooperación al desarrollo como prioridad de la acción exterior. Sin embargo, además de estos elementos, hay que añadir la especial articulación de actores que explican algunos cambios, en el sentido planteado por Sabatier (1993) en referencia a las coaliciones promotoras, incorporándose $\mathrm{ONG}$, entidades locales y fundaciones a la definición y gestión de esta política.

El hecho de que la política de cooperación al desarrollo se elevara en el nivel de priorización por parte del gobierno de José Luis Rodríguez Zapatero de 2004 y 2008, reflejado, entre otras consecuencias, en el significativo incremento presupuestario de esta política, explica, obviamente, que las posibilidades de marcar posiciones de las instituciones con mayores competencias y capacidades de decisión en este ámbito se incrementaron en igual proporción. Este elemento explica por si solo el hecho de que la AECID adquiriera una voz propia en la definición y desarrollo de la política de cooperación en este sector, respondiendo así a una voluntad clara y contundente por parte de sus responsables políticos. Además, este cambio se vio facilitado por algunos otros factores, como el reforzamiento de las estructuras de la AECID en los países destinatarios de la ayuda antes mencionada y la desvinculación teórica y práctica de la política de cooperación con la política exterior, que se reflejó en una mayor autonomía de las OTC y en una limitación de las posibilidades de acción de las Embajadas en materia de cooperación al desarrollo ${ }^{10}$; el propio refuerzo de la estructura de gestión de la AECID en Madrid. Obviamente este empoderamiento de la AECID con respecto a instituciones que hasta entonces habían definido, moldeado, diseñado y gestionado la política en este sector, generó conflictos con algunas instituciones, por ejemplo, con el CGPJ o con el Ministerio del Interior. En cualquier caso, se podría decir que este posicionamiento resultó quizá excesivo en tanto en muchos países, regiones, proyectos y programas se limitó de forma muy clara la presencia de estas entidades públicas, erosionando de paso su capacidad de diálogo e interlocución. Probablemente, el interés por eliminar nichos de decisión a quien no tenía la competencia en política de cooperación al desarrollo (ministerios, poderes públicos o cuerpos profesionales), se llevó por delante las ventajas de oportunidad que podrían extraerse de la relación y la interlocución técnica desarrollada por estas instituciones. A ello hay que sumarle el menor interés en

10 Un punto de inflexión en este aspecto lo constituye la decisión de "eliminar" los consejeros de cooperación en las Embajadas, decisión que generó enorme polémica en el servicio exterior, pero que apuntaló de forma contundente la desvinculación entre ambas políticas. 
términos de política interna que suponían estas entidades, en comparación con lo que podría implicar, por ejemplo, contar con otros socios, como el sector de las ONGD, de las CCAA o de los EELL. Mucho menos exitoso fue la adquisición de voz frente a otros actores que asumieron un papel crecientemente relevante en este sector, como la FIIAPP, que fue ganando progresivamente un rol que sobrepasaba indudablemente los fines y objetivos para los que fue creada, o algunos organismos multilaterales hacia los que se canalizó en parte esta cooperación, como el PNUD, la SEGIB y otros organismos multilaterales.

Además, en la gestión de la política de cooperación al desarrollo en el sector de gobernabilidad democrática, al igual en la gestión de otros sectores, se incorporaron nuevas instituciones y actores públicos y privados, lo que fue facilitado sin duda por el incremento presupuestario, que permitió incorporar nuevos actores sin sacrificar la participación de los tradicionales. De esta forma, a través de los programas y proyectos, se facilitó la relación con actores e instituciones procedentes del sector privado y/o público.

Consecuentemente con esta posición empoderada frente a los socios tradicionales, y en consistencia con el peso político de los responsables de la política de cooperación al desarrollo, resultó sencillo incorporar nuevos actores e instituciones al sector de la gobernabilidad democrática, tanto en lo que se refiere a instituciones públicas como privadas o semipúblicas (Colegios de profesionales, fundaciones de partidos, entes locales, etc.), facilitando a través de la gestión de estos programas las relaciones con todos ellos. Por tanto, la gestión de proyectos y programas se hizo más compleja, poniéndose de manifiesto la necesidad de una estrategia adecuada de articulación y coordinación de actores y socios ${ }^{11}$.

Finalmente, la voluntad por estandarizar la política de ayuda al desarrollo con la llevada a cabo por otros donantes explica en buena medida la necesidad de adaptar temas a las prioridades marcadas internacionalmente (la lucha contra la pobreza) y de incorporar nuevas regiones como destino de la política española, coherente con la incorporación como destino de la ayuda de aquellas regiones marcadas por los altos índices de pobreza (principalmente, África Subsahariana). Por tanto, también derivado de todo lo anterior, resultó coherente incluir nuevos temas y/o reorientar los ya existentes, así como ampliar el trabajo en gobernabilidad democrática a otras zonas geográficas. En el ámbito del apoyo al sistema democrático y a la participación ciudadana, se pusieron en marcha nuevas líneas de trabajo, tomando en cuenta que en muchos países los déficits de gobernabilidad derivan, por una parte, de las carencias en materia de representación política y, por otra, de la debilidad de los pactos políticos sobre los que se debería construir la institucionalidad democrática. Entre estas nuevas líneas se pueden destacar las siguientes: el apoyo a los partidos políticos, como organizaciones que deben canalizar las demandas ciudadanas y convertirlas en opciones políticas12; el apoyo a la concertación y el diálogo social, en tanto que la ausencia de canales de diálogo y comunicación entre actores socioeconómicos dificulta la acción política económica13; el apoyo a las Asambleas Constituyentes, tratando de aportar técnicamente en la construcción de nuevas bases jurídicas sobre las que asentar el pacto político14; o el fortalecimiento de ciertas organizaciones sociales con el fin de que puedan reclamar sus derechos y ejercer control político sobre las instituciones ${ }^{15}$.

En el ámbito del apoyo a la administración pública central, se orientaron y trataron de concentrar casi todas las acciones en la promoción de sistemas de servicio civil profesionalizado y en la capacidad de los gobiernos de planificar y elaborar políticas públicas, especialmente las relativas a la cohesión social ${ }^{16}$.

Por último, en lo que se refiere al apoyo al sector justicia y la seguridad, en lugar de priorizar las intervenciones que enfatizaban en la visión de la justicia como poder político (apoyo a la carrera judicial, fortalecimiento de la Cortes Supremas de Justicia, o creación y/o fortalecimiento de Escuelas Judiciales, entre otros), las intervenciones trataron de llevarse a cabo desde la concepción de la justicia como servicio público al ciudadano y como garante de la protección y defensa de los derechos fundamentales de los ciudadanos. Se potenciaron programas de acceso a la justicia, de agilización de la administración de justicia y se incorporaron nuevos actores, como el Ministerio de Justicia, la Fiscalía General del Estado, o los colegios de abogados y de registradores, entre otros. En el caso

11 Por ejemplo, en el sector justicia, que ha resultado ser uno de los más difíciles de gestionar, en años precedentes fue el Consejo General del Poder Judicial la institución que gestionaba y nutría, casi en exclusiva, las demandas de asistencias técnicas procedentes de los países en desarrollo. Ahora estas solicitudes se gestionan a través de un esquema variable y no exento de conflicto entre diferentes instituciones españolas competentes en el sector. Evidentemente, se hace necesario asumir un rol claro y definido de coordinación y de colaboración entre instituciones en el sector.

12 Por ejemplo, se apoyaron programas de apoyo a los partidos políticos en América Central, en colaboración con el PNUD y el BID, o de carácter bilateral en Rep. Dominicana, por ejemplo.

13 Una muestra de este tipo de programas puede ser el que se desarrolló en Bolivia o en Brasil en 2006.

14 Por ejemplo, en Bolivia o en Ecuador.

15 Son muy numerosos los ejemplos de este tipo de proyectos: apoyo a las organizaciones de mujeres, de defensa de los derechos humanos, de acceso a la justicia, etc.

16 El Programa de apoyo al Fortalecimiento del servicio civil en Centroamérica o el apoyo a la Función Pública en Colombia son algunos ejemplos de este tipo de proyectos.

GESTIÓN Y ANÁLISIS DE POLÍTICAS PÚBLICAS, Nueva Época, nº 10 julio-diciembre 2013 ISSN: 1989-8991 - DOI: 10.24965/gapp.voi10.10119 
del sector de la seguridad, los cambios fueron, si cabe, de mayor envergadura, por ser este un ámbito en el que la AECID venía desarrollando un papel residual, como facilitador de un modelo de cooperación interinstitucional alejado, la mayor parte de las veces, de la visión del desarrollo. En estos años se realizó un tránsito hacia una cooperación focalizada en la necesidad de proveer a los ciudadanos la necesaria seguridad para el desarrollo humano, generando espacios seguros donde poder ejercer los derechos y desarrollando condiciones para la convivencia pacífica. Este trabajo implicó potenciar el apoyo a políticas preventivas de la violencia y de respaldo a la capacidad de las instituciones públicas para proteger a los ciudadanos de la misma y a combatir la impunidad. Este cambio no estuvo exento de debate acerca de cómo hacer operativo el vínculo entre seguridad y desarrollo y cómo apostar por modelos preventivos sin olvidar la importancia que el control y la lucha contra el delito que tiene cualquier política de seguridad. Como ejemplos, sin duda fue del máximo interés la experiencia de puesta en marcha de la línea de apoyo al sector seguridad en el Programa de Cooperación Regional con Centroamérica, que se diseñó en colaboración con otros actores (PNUD) y llevando al máximo los principios de apropiación y alineamiento con respecto a las políticas regionales desarrolladas en el ámbito del SICA o el caso de Colombia.

\section{UNA PROPUESTA DE MEJORA DEL VÍNCULO ENTRE ESTRATEGIA Y ACCIÓN EN LA POLÍTICA DE COOPERACIÓN AL DESARROLLO EN GOBERNABILIDAD DEMOCRÁTICA}

Como ya se ha apuntado, todas estas transformaciones se llevaron a cabo sin adecuada conexión entre la formulación teórico-estratégica acerca del tipo de ayuda que convenía desarrollar en este sector y las acciones y las prácticas llevadas a cabo, que a su vez pone de manifiesto la dificultad para incorporar en esa estrategia la experiencia acumulada en programas y proyectos de los años previos. Esta necesidad de definición estratégica debería haber tomado en cuenta la posición de partida de la cooperación española, sus activos y el contexto cambiante de la realidad a abordar, tanto en lo que se refiere al contexto político, económico y social de los países a los que se destinó, como del contexto cambiante de la comunidad internacional en el debate de la ayuda al desarrollo, marcado por una ortodoxia que no encaja fácilmente en este sector. Todo el entramado normativo desarrollado no se acompañó de la necesaria planificación operativa, lo que resultó especialmente grave en relación con algunas de las dimensiones sobre las que resulta más ineludible la toma de decisiones, como son la definición de las contrapartes idóneas, de los instrumentos más adecuados o del nivel en el cual incidir. Se plantea una propuesta de elementos que deberían ser incorporados en la definición estratégica de la ayuda en este sector, que se corresponden con aspectos de la política de ayuda en el sector de la gobernabilidad democrática en torno a las que se requiere una toma de posición clara y definida.

\section{Las contrapartes de la ayuda}

Ha habido poca definición estratégica en torno a qué contrapartes podrían resultar más idóneas en cada país: las instituciones públicas o ciertos actores y sectores de la ciudadanía con el fin de empoderarles en la reclamación de sus derechos. El enfoque de derechos, que se constituye en la guía de los últimos Planes Directores y de la Estrategia, apuesta tanto por fortalecer las capacidades de la ciudadanía en la reivindicación y reclamación de sus derechos como en el fortalecimiento de las instituciones para garantizarlos y prestarlos. Bien es verdad que la Estrategia privilegió el fortalecimiento de la sociedad civil frente a la promoción del desarrollo institucional. Sin embargo, no se ha tomado en cuenta la necesidad de combinar ambas estrategias, de forma que, tal como señaló Abramovich (2006), se incorporen en las políticas de desarrollo un enfoque de derechos que obligue al Estado al reconocimiento de los derechos y libertades de los sectores excluidos, o las ideas que desarrolló también Domingo (2006), en el sentido de que el fortalecimiento de la ciudadanía no debe concentrarse solamente en el fortalecimiento de la sociedad civil, sino también en el desarrollo de las instituciones públicas. De hecho, Domingo (2006) plantea que actualmente la inclusión y reconocimiento de derechos se está produciendo más por el fortalecimiento institucional que por medio del fortalecimiento social ${ }^{7}$.

\section{El nivel de intervención}

Siguiendo a Cámara (2003), los proyectos y programas se pueden orientar incidiendo en diversos niveles: nivel sistémico, de sector, organizacional o individual. En el caso de la cooperación española desarrollada por la $A E C I D$ en estos años, se han observado carencias importantes en lo que se refiere a una definición estratégica en torno al nivel en el que convenía incidir prioritariamente, o al menos identificar el equilibrio óptimo en cuanto a los niveles a los que orientar la ayuda. En consistencia con la dispersión de programas y proyectos ya mencionada, se han desarrollado acciones muy diversas en cuanto a los niveles de intervención. En unos casos se priorizaron proyectos orientados a la definición de agendas políticas, acompañados, quizá, de apoyo a instituciones de pla-

17 Este es el caso de algunos países en desarrollo en América Latina, África y Europa del Este que, a través del fortalecimiento de ciertas instituciones clave como las Cortes Supremas de Justicia, están logrando promover una transformación social conocida como “la revolución de los derechos" (Domingo, 2006). 
nificación y/o de diseño de políticas. En muchos otros casos, las intervenciones se centraron en apoyos técnicos puntuales consistentes en proyectos de fortalecimiento de una institución concreta o incluso de una acción específica. Y la formación y capacitación continuó siendo la acción más recurrente en la mayor parte de los programas y proyectos $^{18}$. Generalmente, cada OTC fue eligiendo entre la variada demanda de apoyo técnico aquella que podría resultar más adecuada de acuerdo a los principios generales que establecía el PD y la Estrategia.

Evidentemente, en este déficit se puso en evidencia la debilidad de la relación entre la política de cooperación al desarrollo y la política exterior, por lo que en algunos casos se coordinaron e integraron ambas políticas de manera idónea mientras que en otros se reflejó o bien la todavía instrumentalización de la política de cooperación por parte de la política exterior o bien la descoordinación entre ambas. Bien es verdad que en la mayoría de los casos se impuso el sentido común y la profesionalidad de los técnicos para generar un equilibrio entre ambas posiciones, más allá de las carencias de directrices o de posicionamiento estratégico. En cualquier caso, parece necesario avanzar en la identificación del nivel la incidencia y el impacto de la ayudan pueden ser mayores.

Adicionalmente, se generó una confusión entre la pertinencia de apoyar el ámbito local frente al nacional, en la convicción de que el ámbito local es más democrático y podría ofrecer resultados a más corto plazo, sin tomar en cuenta que el ámbito local es un espacio más de gobierno, sin que necesariamente vaya asociado a mayor democracia o a mayor participación. Descentralización no es sinónimo de participación y mucho menos de democracia.

\section{Los fines políticos de la ayuda}

Este déficit de reflexión se relaciona también con el déficit de reflexión acerca de qué objetivo final se persigue en este sector, desde la convicción de que el apoyo a la gobernabilidad democrática genera impactos distintos dependiendo de si se enfatiza en la idea de gobernabilidad o en la idea de democracia. Mientras la primera idea, derivada del enfoque institucionalista, pone el foco en la necesidad de contar con instituciones fuertes, estables y eficaces para el funcionamiento del mercado y por tanto para generar crecimiento y desarrollo (gobernabilidad democrática como instrumento para el desarrollo), la segunda idea, sustentada en el enfoque de desarrollo humano, concibe la democracia como una dimensión del desarrollo (gobernabilidad democrática como fin).

En este sentido, la reflexión teórico-estratégica quedó muy limitada en lo que se refiere a la posición en torno al apoyo a la democracia, quedándose en definiciones estratégicas más o menos pomposas y grandilocuentes sin mayores precisiones acerca de las dimensiones esenciales relacionadas con la democracia: participación, rendición de cuentas, pluralismo ideológico, desarrollo de libertades y derechos, etc. En general, no se operativizó el vínculo entre democracia y desarrollo, siendo que hay toda una corriente de análisis en las ciencias sociales acerca de las conexiones entre ambas dimensiones, desde los trabajos de Lipset (1959) hasta los más recientes de Maravall (2005) pasado por O'Donnell (1979), Sen (1989 y 1999), North (1991) o Rodrik (1997) $)^{19}$ y sobre todo se obvió el debate entre lucha contra la pobreza como prioridad y apoyo a la democracia como requisito o condición. Desde el momento en que la democracia se considera una dimensión misma del desarrollo humano (PNUD: 2004), pierden relevancia las posiciones que apuestan por la gobernabilidad (sin adjetivo) como medio para promover desarrollo. Este déficit de definición estratégica impidió resolver la forma idónea de trabajo con países con regímenes no democráticos (algunos países africanos, Cuba). Pero además, impidió abordar las implicaciones que tiene el debate actual sobre calidad de la democracia en países en desarrollo. El hecho de que casi todos los países de la región iberoamericana se sitúen en la categoría de democráticos, no resuelve en absoluto cómo incidir y cómo apoyar la construcción de mejores democracias, qué subsectores afectan de forma más directa a la calidad de la misma (representación, rendición de cuentas, transparencia, eficacia, etc.) y sobre todo, cómo inciden estas cuestiones en el principio de condicionalidad de la ayuda ${ }^{20}$, cómo superar las recurrentes tendencias a exportar modelos en este ámbito y qué ámbito marginal queda para el apoyo externo a la democracia. En este último aspecto, existe un debate acerca de las posibilidades de impacto de la ayuda internacional en la mejora de la calidad de la democracia y de sus instituciones. Los análisis de Carothers (1999) o de Santiso (2002) ponen el foco en las formas más eficientes de apoyar la democracia en los países en desarrollo, e identifican las posiciones más pesimistas que plantean que hay poco margen para que actores externos contribuyan a promover democracia en países en desarrollo. Este debate conduce a la cuestión de la eficacia de la ayuda y a las posibilidades de ofrecer resultados en el corto plazo.

18 La relación entre la formación y el fortalecimiento institucional fue magníficamente planteada por Cámara. El PIFTE ha dado lugar también a alguna reflexión en torno a esta relación. Al respecto, ver la Evaluación realizada por la AEVAL en 2009 sobre este Programa.

19 Para una revisión rápida de esta relación ver Rocha-Menocal (2010).

20 Esta cuestión adquiere toda su relevancia en aspectos tales como la incorporación de algunos de los nuevos instrumentos, como el apoyo presupuestario. 


\section{El modelo de oferta}

Y finalmente, ha habido poca definición en cuanto al equilibrio óptimo entre oferta y demanda de la ayuda española en este sector. No ha habido mucha reflexión sobre lo que significa la oferta de cooperación española, qué esperan del apoyo español los países socios, o sobre qué subsector tiene mejores condiciones o supone una ventaja añadida en relación a otros. En resumen, ha faltado orientación estratégica en torno a la forma idónea para que la cooperación española pueda resultar catalítica en la generación de procesos de cambio. Tampoco se ha desarrollado adecuadamente la reflexión en lo que se refiere a la definición de la demanda, más allá de las ventajas de ciertos instrumentos, como las comisiones mixtas o los Marcos de Asociación (MAE), que sin duda han supuesto un importante avance en este ámbito. Siguen pendientes debates importantes, como las posibilidades de la estandarización de este tipo de cooperación, la conveniencia de llevar el principio de concentración hasta el final en este sector ${ }^{21}$, la exageración del papel del donante o su capacidad para convertirse en un actor interno, sobre todo en países muy dependientes de la ayuda externa, y por tanto, en su posible instrumentalización política o en los efectos perversos que como tal actor puede acabar generando en el país destino de esta ayuda.

\section{Los instrumentos de la ayuda}

En lo que se refiere a los instrumentos de la cooperación, resulta especialmente necesario llevar a cabo una reflexión acerca de los nuevos instrumentos. El debate sobre la cooperación con países de renta media ha puesto sobre el tapete la necesidad de ir más allá de la forma tradicional de prestar la ayuda, basada en el formato proyecto, que implicaba que además de aportar los recursos económicos, se trasladaban también los recursos técnicos y con ellos el modelo político implícito a esta panoplia de posibilidades técnicas. El debate de los PRM ha puesto de manifiesto la centralidad de las instituciones para el desarrollo y por tanto la potencialidad de instrumentos que superan la visión donante-receptor, y que asumen la existencia de islas de excelencia técnica y de conocimiento que no se encuentran distribuidas de forma uniforme. Por tanto, la cooperación sur-sur o regional adquiere toda su potencia en relación a este sector, en el que resulta especialmente útil y necesario acceder al conocimiento de experiencias y soluciones técnicas, estén donde estén. También la cooperación triangular adquiere creciente interés, así como la cooperación regional, como espacio no solo para generar sinergias sino para abrir ventanas de oportunidad a procesos de cambio que difícilmente podrían darse en el plano nacional, constituye sin duda una modalidad a potenciar en el futuro.

\section{CONCLUSIONES}

Teniendo en cuenta esta trayectoria y los datos aportados, se podría concluir que la política de cooperación al desarrollo en materia de gobernabilidad democrática ha mantenido algunos de sus rasgos distintivos en esta etapa de profundas transformaciones generales, como son la orientación a la demanda planteada por los países receptores de la ayuda, el peso de las administraciones públicas en la gestión de este sector, sobre todo en la identificación de proyectos, la excesiva dispersión de proyectos y actividades que componen el sector y la orientación prioritaria hacia América Latina.

Sin embargo, estas características no se han integrado de forma adecuada en una programación sectorial que orientara y definiera estratégicamente el sector. La trayectoria y la experiencia acumulada en este sector, que otorgaron una serie de rasgos identitarios, no se utilizaron como insumo decisivo para definir adecuadamente una política de cooperación en este sector, que sumara la valiosa y poco evaluada trayectoria española en este ámbito a definiciones estratégicas acordes con los debates internacionales en la materia. Antes al contrario, en el mejor de los casos se obvió la trayectoria de la cooperación española en este sector, cuando no se desprestigió directamente; evaluación negativa a la que se llegó por intuición ante la carencia de suficientes evaluaciones en este sector.

En este sentido, se perdió una oportunidad única de dotar a este sector de una clara y contundente definición estratégica, sumando los aspectos valiosos de la experiencia acumulada en este sector al impulso de renovación derivado de la apuesta por modernizar la cooperación española en general. Y esta necesidad de definición estratégica en este sector sigue siendo especialmente necesaria en estos momentos, ya que el resto de la comunidad internacional reconoce a España un valor añadido precisamente por su experiencia en el sector de la goberna-

21 Este es un sector que consume poco recursos. Quizá para desarrollar una cooperación realmente catalítica sea conveniente revisar el principio de concentración y el vínculo con otras políticas, especialmente la exterior. 
bilidad democrática, especialmente orientada hacia los países de renta media. En las actuales circunstancias, de cambios en la arquitectura de la ayuda, con la aparición de nuevos actores, nuevos esquemas, nuevas modalidades y nuevos desafíos, se hace imprescindible definir estratégicamente el apoyo que España pueda brindar al fortalecimiento de las instituciones democráticas y a la gobernabilidad democrática.

\section{BIBLIOGRAFÍA}

ABRAMOVICH, Víctor (2007) “Una aproximación al enfoque de derechos en las estrategias y políticas de desarrollo" en: ERAzO, Ximena (Coord.) Políticas públicas para un estado social de derechos: el paradigma de los derechos universales, Ed. LOM, pp. 91-118.

AGENCIA ESPAÑola de COOPERACIÓn INTERNACIONAL PARA el DeSARROLLo (AECID) (2009) Diagnóstico de la Ayuda de la AECID en el sector de gobierno y sociedad civil (2005-2008)

Alonso, José Antonio (2007) “¿Es necesario ayudar a los países de renta media?” En: IGLESIA CARUNCHO, Manuel (coord.) y otros. Avances y retos de la cooperación española: reflexiones para una década, Fundación Carolina y Siglo XXI Editores, Madrid, pp. 157-170.

AyUso, Anna (2007) "De París 2005 a Accra 2008: el compromiso con los resultados". En: IGLESIA CARUNCHO, Manuel (coord.) y otros. Avances y retos de la cooperación española: reflexiones para una década Fundación Carolina y Siglo XXI Editores, Madrid, pp. 565-582.

CÁmARA, Luis (2003). La cooperación técnica española para el refuerzo institucional. Desarrollo humano e institucional en América Latina (DHIAL), vol. 38, num. 1.

CAPANO, G. y HowletT, M. (2009) “The Multidimensional World of Policy Dynamics". En: CAPANo, G. y HowletT, M. (eds.) European and North American Policy Change: Drivers and Dynamics ( $1^{\mathrm{a}}$ Ed., 1-12): Routledge ECPR Studies in European Political Science.

CAROTHERS, Thomas (1999). Aiding Democracy abroad: the Learning Curve. Carnegie Endowment.

Development Assistance Committee (DAC) (2011) Peer Review 2011, Organization for Economic Cooperation and Development.

DIAMOND, Larry. (1993) “Reconsideración del nexo entre desarrollo económica y democracia”. En: Centro de Estudios Públicos, n 49, Santiago de Chile.

Díaz-RATO, Aurora (2007) "La cooperación española en América Latina". En: IGLESIA CARUNCHO, Manuel (coord.) y otros. Avances y retos de la cooperación española: reflexiones para una década, Fundación Carolina y Siglo XXI Editores, Madrid, pp. 349-362.

DIRECCIÓN DE COOPERACIÓN CON AMÉRICA LATINA (DCALC) Agencia Española de Cooperación Internacional para el Desarrollo (AECID) (2012) Informe sobre las intervenciones de cooperación en los sectores de gobernabilidad democrática y construcción de paz en América Latina (2008-2011)

GarGarella, Roberto, Domingo, Pilar, y Roux, T. (Eds.). (2006). Courts and Social Transformation in New Democracies: an institutional voice for the poor? Ashgate Publishing Company, London.

GRINDLE, Merilee (2007) “Good Enough Governance Revisited”. Development Policy Review 25(5): 553-574.

HoGwood, Brian W. y Peters, Guy (1983) Policy Dynamics. Harvester Wheatsheaf, 1983.

IGLESIA CARUNCHO, Manuel (coord.) y otros (2007) “Aproximación a una visión de conjunto”. En: IGLESIA CARUNCHO, Manuel (coord.) y otros. Avances y retos de la cooperación española: reflexiones para una década, Fundación Carolina y Siglo XXI Editores, Madrid, pp. 3-38.

Kaufmann, Daniel, KraAy, Aart y Zoido, Pablo (1999) "Governance Matters". Policy Research Working Papers 2196. The World Bank, Washington, D.C.

LINDBLOM, Charles E. (1992): “La ciencia de salir del paso" y "Todavía tratando de salir del paso". En: AGUILAR, L. (ed.) La hechura de las políticas, México: Porrua, 201-254.

LIPSET, Seymour Martin (1959) "Some social requisites of democracy: Economic development and political legitimacy “. En: The American Political Science Review, 53(1), 69-105.

MARAVALL, José María (2005) El control de los políticos, Taurus Ediciones, 2003.

MinTZBERG, Henry y JORGENSEN, Jan (1995) “Una estrategia emergente para la política pública”. En: Gestión y 
Política Pública, vol. IV número 1, primer semestre, pp. 25-46.

NORTH, Douglass

- (1990) Institutions, Institutional Change and Economic Development. Cambridge University Press. Cambridge, Massachusetts.

- (1991) “Institutions”. En The Journal of Economic Perspectives, 5 (1), pp. 97-112.

O’DONNELL (1994) “Delegative Democracy”, Journal of Democracy, Vol. 5, No. 1, January 1994: 55-69, National Endowment for Democracy and The Johns Hopkins University Press.

PRZEWORSKI, Adam (2004) “Democracy and Economic Development.” En: Edward D. MANSFIELD y Richard SISSON (eds.), The Evolution of Political Knowledge. Ohio State University Press, Columbus.

ROBLES, Gonzalo (2007) “La cooperación española al desarrollo”. En: IGLESIA CARUNCHO, Manuel (coord.) y otros. Avances y retos de la cooperación española: reflexiones para una década, Fundación Carolina y Siglo XXI Editores, Madrid, págs. 73-82.

RochA MenOCAL, Alicia (2010) "Analysing the relationship between democracy and development: defining basic concepts and assessing key linkages", ODI Papers, Londres.

Rodrik, Dani, Arvind SUbramaniAn y Francesco TrebBI (2004) “Institutions Rule: the Primacy of Institutions over Geography and Integration in Economic Development”. Journal of Economic Growth 9, 131-165.

RODRIK, Dani. (1997). Has globalization gone too far? Peterson Institute.

Sabatier, Paul A. (1999) Theories of the Policy Process, Westview Press.

SABAtier, Paul A. Y Jenkins-Smith, Hank C. (1993) Policy change and learning: an advocacy coalition approach". Westview Press.

SANTISO, Carlos (2002). "Retos y desafíos de la reforma de la ayuda europea. Mejorando las respuestas de la Unión Europea a las crisis de gobernabilidad y las erosiones de la democracia”. En: Revista Instituciones y DesarroIlo, núm. 11, pp. 7-62.

Seguimiento Plan Anual de Cooperación (PACI) (2007), Secretaría de Estado de Cooperación Internacional.

Seguimiento Plan Anual de Cooperación (PACI) (2008), Secretaría de Estado de Cooperación Internacional.

Seguimiento Plan Anual de Cooperación (PACI) (2009), Secretaría de Estado de Cooperación Internacional.

Seguimiento Plan Anual de Cooperación (PACI) (2010), Secretaría de Estado de Cooperación Internacional.

Seguimiento Plan Anual de Cooperación (PACI) (2011), Secretaría de Estado de Cooperación Internacional.

Seguimiento Plan Anual de Cooperación (PACI) (2012), Secretaría de Estado de Cooperación Internacional y para Iberoamérica.

SEN, Amartya

- (1989) Sobre ética y economía, Alianza Editorial, Madrid.

- (1999) "Ética del comportamiento y éxito económico" en Revista de Occidente, № 215, 1999, pp. 123-137. 